\title{
AGENTES DE ONICOMICOSIS EN MANOS Y PIES EN VALDIVIA (CHILE)
}

\section{Onichomycosis agents in hands and feet in Valdivia(Chile)}

\author{
Zaror L. ${ }^{1}$, Moreno M.I. ${ }^{2}$, Vega $\mathbb{K}^{3}$, Hering M. ${ }^{2}$ y Frick . $^{2}$ \\ ${ }^{1}$ Instituto Microbiología Clínica \\ ${ }^{2}$ Instituto de Especialidades (Departamento Dermatologia), \\ ${ }^{3}$ Escuela de Tecnología Médica, \\ Universidad Austral de Chile. \\ Casilla 567, Valdivia, Chile
}

Palabras clave: Dermatofitos, levaduras, onicomicosis.

Key words: Dermatophytes, yeast, onichomycosis.

\section{RESUMEN}

Se estudiaron 78 muestras de uñas de manos y pies de 74 pacientes con diagnóstico de onimicosis

Para la obtención de la muestra se raspó la lámina interna de la uña, excepcionalmente la lámina externa y se efectuó el examen microscopico directo ya sea en $\mathrm{KOH}-20 \%$ - tinta Parker 51 azul permanente o con KOH-20\% - Chloroback $E$. Todas las muestras fueron sembradas en agar Sabouraud dextrosado, agar Lactrimel y agar selectivo para dermatofitos (DIM) e incubadas a $28^{\circ} \mathrm{C}$ por 10-15 dias.

De las muestras estudiadas, en el 79,5\% se observaron hongos al examen microscópico directo, aislándose estos en el $61,5 \%$ de los cultivos.

La frecuencia de agentes de onimicosis fue: $\boldsymbol{C}$.albicans $(43,75 \%), \quad$ T. rubrum $(31,25 \%)$ y T.mentagrophytes (10,42\%). C. albicans se aisló en el 70,8\% de las onimicosis en manos de mujeres.

T.rubrum se aisló en un 50 y 25\% de hombres y mujeres respectivamente. En pie se aisló en el 52,9\% y en mano en un $19,3 \%$.

S. brevicaulis fue aislado en un solo caso.

\section{INTRODUCCION}

Las patologías ungueales, pueden tener distintas etiologías micóticas, bacterianas, traumáticas, peládicas o estar asociadas a otros procesos patológicos, como : alopecía areata, afecciones clínicas generales, alteraciones genéticas u otras(Reddy et al.,1977; Zaror et al., 1983).La onicomicosis es una de las principales onicopatías en el hombre, observada por lo general en personas mayores de 14 años (Zaror, 1974, 1979).

El comienzo es lento e insidioso, en ocasiones

\section{SUMMARY}

Seventy eight samples of hand and foot nails from patients with a onichomycosis diagnosis were studied

Samples were obtained by scraping inner lamina of the nail, and exceptionally the external lamina. A direct microscopic examination was carried out with $\mathrm{KOH}-20 \%$ Parker 51 permanent blue ink, or KOH-20\% ChlorobackE. Every sample was sowed in agar Sabouroud dextrose, agar Lactrimel and in the selective agar for dermatophytes (DIM) and incubated at $28^{\circ} \mathrm{C}$ for 10-15 days

Of the samples studied the fungi was observed in the $79,5 \%$ under a direct microscopic examination, and it was isolated in the $61,5 \%$ of the cultures.

C.albicans and T.rubrum were isolated in the 43,75\% and $31,25 \%$ respectively where as $\boldsymbol{T}$. mentagrophytes in the $10,42 \%$. C. albicans was isolated in a $70,8 \%$ on hand onychomicosis in women.

T.rubrum was isolated in a 50 and $25 \%$ in men and women respectively. In the foot it was isolated in a 52,9\% and in a 19,3\% in the hands. S. brevicaulis was isolated only in one case.

están afectadas todas las uñas (Rodríguez et al.,1993). Sus principales agentes son especies de los géneros Trichophyton y Candida (De Castro et al.,1983; Zaror, 1982-1983; Rodríguez et al.,1993). Las especies, más aisladas son: Trichophyton rubrum, Candida albicans, Trichophyton mentagrophytes(Achten \& Wanet-Rouard, 1978; Zaror y et al., 1983; Rosas \& Zaror 1987). Otras especies aisladas son: T .tonsurans (De Castro et al.,1983), Epidermophyton floccosum y Trichophyton violaceum (Achten y WanetRouard 1978). Rodríguez et al., 1993 describen a Candida parapsilosis como la especie más aislada del género. Otra 
especie más frecuente que $C$. albicans en las uñas de los pies sería según Williemsem (1933). Candida guilliermondi.

Eventualmente se observan casos de micosis causadas por hongos filamentosos del tipo oportunista, pertenecientes a los Hyphomyceyes, tales como: Scopulariopsis spp., Aspergillus spp. y Fusarium spp. (Zaias 1972: Achten el al., 1979; Zaror. 1979; Zaror \& Frick 1980; Negroni 1984). por Basidiomycetes (Kligman 1950) y por Ascomycetes tales como Botryodiplodia theobromae (Restrepo et al., 1976).

Tabla 1.- Distribución de la localización de la lesión ungueal según sexo, en pacientes sospechosos de onicomicosis en Valdivia

\begin{tabular}{|l|rc|rc|rr|}
\hline \multicolumn{9}{|c}{ Masculino } & \multicolumn{2}{c|}{ Femenino } & \multicolumn{2}{c|}{ Total } \\
\hline Localizac. & $\mathrm{n}$ & $\%$ & $\mathrm{n}$ & $\%$ & $\mathrm{n}$ & $\%$ \\
& & & & & & \\
Manos & 9 & 49,9 & 27 & 51,9 & 36 & 48,7 \\
Pies & 12 & 54,5 & 22 & 42,3 & 34 & 45,9 \\
Manos y & 1 & 4,6 & 3 & 5,8 & 4 & 5,4 \\
pies & & & & & & \\
\hline Total & 22 & 100,0 & 52 & 100,0 & 74 & 100,0 \\
\hline
\end{tabular}

\section{Pauta General de Trabajo}
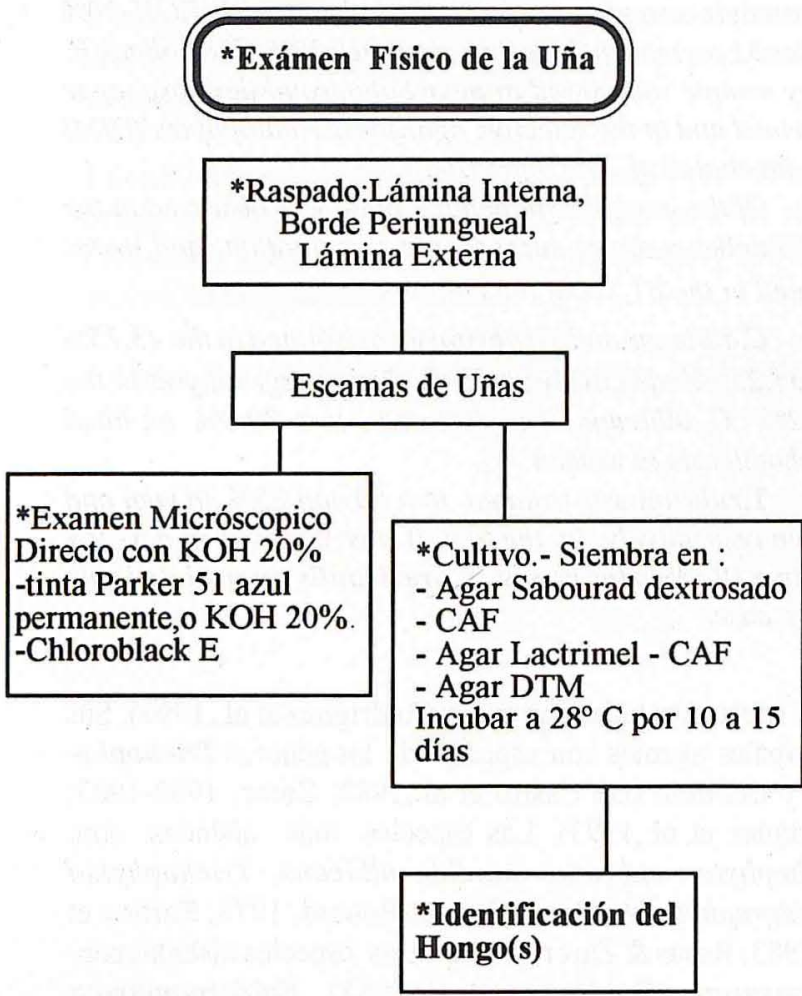

Para considerar a estos como responsables de una onicomicosis de tipo oportunista se deben seguir los criterios de Achten y Wanet-Rourd (1978).

Las onicomicosis de los pies son producidas en su mayor parte por dermotrofitos y afectan de manera similar a hombres y mujeres. Al contrario, las onicomicosis de las manos son más frecuentes en mujeres y las especies más aisladas pertenecen al género Candida. (Achten \& WanetRouard 1978 (13): Zaror et al., 1982: Williemsem, 1993).

Las onicomicosis se clasifican en 4 tipos (Zaías, 1972; modificada por Zaror, 1983): subunguel distal, superficial blanca, subungueal proximal, candidiásica periungueal y candidiásica tricofitoide. Esta clasificación es útil en el diagnóstico clínico incial como también en el momento de obtención de la muestra.

De acuerdo a los antecedentes previos, nuestros objetivos fueron: determinar los agentes de onicomicosis en Valdivia (en manos y pies), según edad de los pacientes, ubicación de las lesiones, su clasificación (Zaias modificada por Zaror), correlación y rendimiento directo cultivo y correlación clínico-micológica.

\section{MATERIALES Y METODOS}

Se analizaron 78 muestras de uñas obtenidas de 74 pacientes con alteraciones unguelas ( 52 de mujeres y 22 de hombres), enviados por el Servicio de Dermatología y E.T.S., otros servicios del Hospital Base de Valdivia y privados, durante Agosto a Diciembre de 1993. Se realizó un examen físico de las uñas, registrando todos los antecedentes en una ficha micológica, confeccionada para el estudio.

Las muestras se tomaron mediante el raspado de la lámina interna de la uña, del borde periungueal $\mathrm{y}$, ocasionalmente, de la lámina externa, con un bisturí; el día cero, el día 30, al final del tratamiento y un mes posttratamiento (como parte de otro estudio). Las muestras se colocaron en portaobjetos esterilizados por flameado. Para realizar el examen microscópico directo se empleó la técnica de aclaramiento con hidróxido de potasio al $20 \%$ con tinta Parker 51 azul permanente; y eventualmente hidróxido de potasio al $20 \%$ adicionado de Chloroblack E (Burke, 1984). La totalidad de las muestras fueron sembradas en agar Sabouraud dextrosado, agar Lactrimel (ambosadicionados de cloramfenicol levógiro) y agar selectivo para dermatofitos (DIM). Las muestras fueron incubadas a $28^{\circ} \mathrm{C}$ por un tiempo promedio de 10 a 15 días. Para la identificación de los dermatofitos se utilizaron las claves de Rebell \& Taplin (1970), de Lodder (1970), para hongos levaduriformes no productores de túbulo germinativo y para Candida albicans la prueba Reynolds \& Braude (1956).

\section{RESULTADOS}

Las lesiones en las uñas de las manos son más frecuentes en mujeres y en los hombres en las de los pies 
Tabla 2.- Exámen Micróscopico directo y cultivo de 78 muestras ungueales, según edad.

\begin{tabular}{|c|c|c|c|c|c|c|c|c|c|}
\hline \multicolumn{5}{|c|}{ Examen Directo } & \multicolumn{3}{|c|}{ Cultivo } & & \\
\hline $\begin{array}{l}\text { Edad } \\
\text { (Años) }\end{array}$ & $\begin{array}{l}\text { Po } \\
\text { n }\end{array}$ & $\%$ & $\underset{n}{\text { Negativo }}$ & $\%$ & $\begin{array}{c}\text { Positivo } \\
\text { n }\end{array}$ & $\%$ & $\begin{array}{c}\text { Negativo } \\
n\end{array}$ & $\%$ & Total \\
\hline $0-15$ & 6 & 60.0 & 4 & 40.0 & 5 & 50.0 & 5 & 50.0 & 10 \\
\hline $16-30$ & 9 & 69.2 & 4 & 30.0 & 7 & 53.8 & 6 & 46.2 & 13 \\
\hline $31-45$ & 22 & 88.0 & 3 & 22.0 & 19 & 76,0 & 6 & 24,0 & 25 \\
\hline 46 y más & 25 & 83,3 & 5 & 16,7 & 17 & 56,7 & 13 & 43,3 & 30 \\
\hline Total & 62 & 79,5 & 16 & 20,5 & 48 & 61,5 & 30 & 39,7 & 78 \\
\hline
\end{tabular}

(Tabla 1). En la distribución de las onicomicosis según las edades de los pacientes, versus el examen microscópico directo y cultivo, se observó que: en un $61,5 \%$ de los cultivos se aisló el hongo, el cual se visualizó en el 79,5\% de los exámenes microscópicos directos. El rendimiento del cultivo versus examen directo fue de un $74,4 \%$ y se observó que, el mayor número de muestras positivas se encuentra en personas mayores de 30 años de edad. En el intervalo de 0-15 años se presentó el menor porcentaje de onicomicosis (Tabla 2).

Los principales agentes de onicomicosis fueron C. albicans $(43,75 \%)$, T. rubrum $(31,25 \%)$ y T.mentagrophytes (10,42\%); la asociación de Candida y T.rubrum fue de $4,17 \%$ y las especies del género Candida comprometieron más de la mitad de la casuística $(52,08 \%)($ Tabla 3$)$.

Tabla 3.- Onicomicosis según su agente causal

\begin{tabular}{|l|r|r|}
\hline & \multicolumn{2}{c|}{ Casos } \\
\hline Agente Causal & Número & $\%$ \\
\hline Trichophyton rubrum & 15 & 31,25 \\
T. mentagrophytes & 5 & 10,42 \\
T. rubrum y C. albicans & 2 & 4,70 \\
C. albicans & 21 & 43,75 \\
C. parapsilosis & 3 & 6,25 \\
C. guilliermondii & 1 & 2,08 \\
Scopulariopsis brevicaulis & 1 & 2,08 \\
\hline TOTAL & 48 & 100,0 \\
\hline
\end{tabular}

La incidencia de onicomicosis según sexo permitió comprobar que de cada 100 personas con algún tipo de onicopatía, en el $64,9 \%$ es causada por hongos ya sea levaduriformes o filamentosos (Tabla 4).
Tabla 4. Incidencia de onicomicosis según sexo

\begin{tabular}{|l|rr|c|}
\hline \multirow{2}{*}{ Sexo } & \multicolumn{2}{|c|}{ Casos } & \multicolumn{1}{c|}{$\begin{array}{c}\text { Tasa de incid. de } \\
\text { onicomicosis } \\
\text { (x100) }\end{array}$} \\
\cline { 2 - 3 } & $\mathbf{n}$ & $\mathbf{\%}$ & \multicolumn{1}{|c|}{54,5} \\
Masculino & 12 & 25,0 & 69,2 \\
Femenino & 36 & 75,0 & 64,9 \\
Total & 48 & 100,0 & \\
\hline
\end{tabular}

La distribución de los hongos aislados según sexo y localización demostró que $C$. albicans se aisló en el $70,8 \%$ de las onicomicosis desde las manos de mujeres (Tabla 5). Los tipos de lesiones ungueales más observadas fueron las subungueales-distales con un 39,6\% (Tabla 6).

\section{DISCUSION}

Los principales agentes de onicomicosis detectados con mayor frecuencia en Valdivia ( C. albicans, T.rubrum y T. mentagrophytes) coinciden con lo observadoen la literatura. (Zaias, 1972; Achten \& Wanet-Rourard, 1978; Zaror 1979; Mc Aleer, 1981; De Castro et al., 1983; Zaror et al., 1983; Rosas \& Zaror, 1987).

Según Rodríguez et al.(1983), del total de onicopatías que afectan al hombre, la onicomicosis representa el $78,7 \%$, lo cual concuerda con nuestros hallazgos (Tabla 4). A su vez, Achten \& Wanet-Rouard (1978) y Williemsem (1993), muestran que las onicomicosis afectan a un 30-40\% de la población en general.

En el presente trabajo se observa que las mujeres son más suceptibles a las onicomicosis que los hombres, coincidiendo con lo postulado por Prochacki \& Bielunska (1967). En nuestro estudio esto sólo ocurriría en las candidosis, pues no se observa una clara diferencia en la tricofitosis. La mayor frecuencia de candidosis de manos en las mujeres (87,5\%), se debería según Achten et al., 1979 y Mc Aleer (1981), al reiterado contacto de las manos con agua, detergentes y azúcares, además del traumatismo que produce el manicure. El principal agente causal sigue siendo $C$. albicans (Zaror, 1982; Zaror et al., 1983; De Castro et al., 
1983; Rosas \& Zaror, 1987); hecho que comprobamos nuevamente en este estudio.

En nuestros resultados se puede apreciar una pequeña diferencia en la distribución de la localización de la lesión ungueal con respecto al sexo. La lesión del pie es levemente más frecuente en hombres $(54,5 \%)$ y la lesión de la mano lo es en mujeres $(51,9 \%)$. Las sospechas clínicas de onicomicosis se comprobaron en el $79,5 \%$ de los exámenes microscópicos directosy en el $61,5 \%$ de los cultivos realizados. $\mathrm{El}$ alto porcentaje de muestras positivas al examen directo indica una buena sospecha clínica,lo que permite que se establezca en forma rápida su diagnóstico y orienta acerca de la conducta terapéutica. El bajo rendimiento del cultivo versus el examen directo ( Tabla 3 ), pudo deberse a 3 factores:

a)En que los elementos del hongo observado al examen directo sean formas muertas localizadas en la parte distal de la uña (causa citada por English ,1973)

b) según Badillet et al.(1981); Zaror et al., (1983); Piontelli \& Toro (1987), es la existencia de hongos saprotrofos contaminantes en el detritus subungueal en el que se pueden almacenar y eventualmente desarrollar. observa la muestra.

c) Inexperiencia de la persona que toma y

En dos mujeres se encontró la asociación de $T$. rubrum y C. albicans; uno en pie y otro en mano. Zaror et al. (1983) y Rosas \& Zaror (1987), han reportado numerosas asociaciones. La presencia de levaduras desde las manos de mujeres, tales como $C$. parapsilosis y $C$. guilliermondii; $(6,3 \%$ y $2,1 \%)$, se han reportado en la literatura, Mc Aleer (1981), las encuentran en $24,6 \%$ y $5,3 \%$ respectivamente. Williemsem (1993) y Rodríguez (1993), describen a estas especies como las más aisladas del género Candida, inclusive más frecuentes que $\boldsymbol{C}$. albicans.

Se comprobó sólo un caso de onicomicosis oportunista por hongo filamentoso, causada por Scopulariopsis brevicaulis. Los porcentajes de onicomicosis de tipo
Tabla 6. Onicomicosis según tipo de lesión y agente

\begin{tabular}{|c|c|c|}
\hline $\begin{array}{l}\text { Tipo de Agente Aislado } \\
\text { lesión }\end{array}$ & N & $\%$ \\
\hline T.rubrum & 10 & 20.0 \\
\hline T. mentagrophytes & 4 & 8,3 \\
\hline T.rubrum y $C$ albicans & 2 & 4,2 \\
\hline S. brevicaulis & 1 & 2,1 \\
\hline C. albicans & 2 & 4,2 \\
\hline C.albicans & 2 & 4,2 \\
\hline T.rubrum & 3 & 6,2 \\
\hline T.mentagrophytes & 1 & 2,1 \\
\hline C.albicans & 5 & 10,6 \\
\hline C.parapsilosis & 1 & 2,1 \\
\hline C. Guilliermondii & 1 & 2,1 \\
\hline C.albicans & 10 & 20,8 \\
\hline T.rubrum & 2 & 4,2 \\
\hline C.parapsilosis & 2 & 4,2 \\
\hline C.albicans & 2 & 4,2 \\
\hline Total & 40 & 100,3 \\
\hline
\end{tabular}

S-D: Subungueal Distal; S-P: Subungueal Proximal; S-B: Superficie Blanca; C-T: Candidiásica Tricofitoide; C-P: Candidiásica Periungueal.

oportunista son variables. Achten (1978), encontró un 2\%., mientras otro estudio, revela que entre un 6$14 \%$ de las micosis ungueales serían causadas por hongos oportunistas. Achten et al. (1979) y Zaror et al. (1983), describen un porcentaje de un $15.8 \%$. El 2,1\% encontrado en nuestro estudio concuerda con lo reportado en la literatura, $S$. brevicaulis es el agente oportunista más aislado en este tipo

Tabla 5. Hongos aislados según sexo y localización en lesiones ungueales

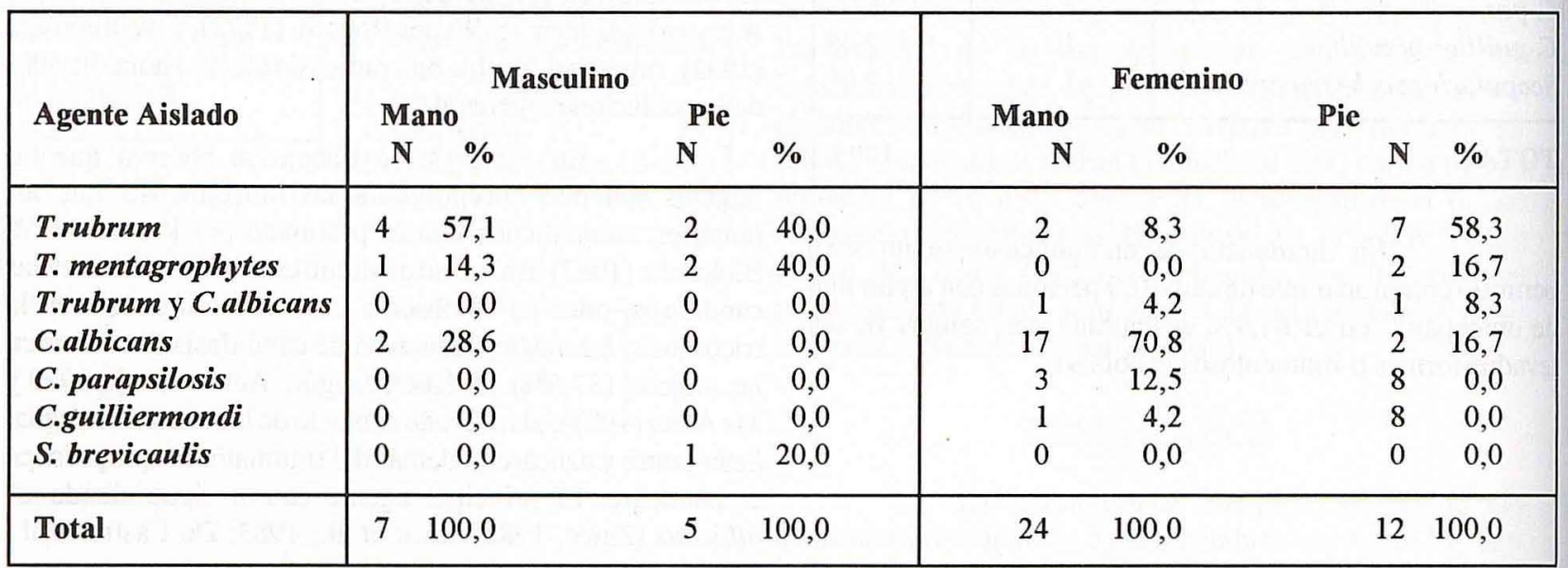


de micosis ungueal (14) (Achten et al., 1979); Zaror et al.,1980, 1982, Piontelli \& Toro 1988).

Las infecciones ungueales distales son las más frecuentes (39,6\%), existiendo predominio de $T$. rubrum y $T$. mentagrophytes. Por otra parte, las lesiones subungueales proximales fueron escasas $(4,25)$. Las micosis superficiales blancas se presentaron en un 8,3\% y las por Candida en un $35,4 \%$. Estas observaciones concuerdan con otras obtenidas por Zaias (1972) y Zaror et al., (1983).

\section{REFERENCIAS}

1.-Achten, G.\& Wanet-Rouard.(1978). Onychomycoses in the laboratory. Mykosen, Suppl. 1: 125-127

2.- Achte, G.; Wanet-Rouard , J.; Wiame, L.; Van Hoof, F.(1979). Onychomycoses a Moisissures. Dermatológica 159: Suppl. 1: 128-140.

3.- Achten, G. (1978).De l'ongle normal a l'ongle pathologique. Cutis 2:411433

4.- Badillet, G.; Pangiotidow, D.; Cabral ,O.(1981). Ungueal dermatophytoses without clinical manifestations. Bull de la Soc. Franc. Myc. Med. 10:213217

5.- Burke, W. \& Jones, B. A. (1984).A simple stain for rapid office diagnosis of fungus infections of the skin. Archives of Dermatology, 120:1519-1520

6.-De Castro, E..; Fischman , O.; Baptista, G.; Flavio, L.(1983). estudio micológico e clínico de 102 casos de onicopatías. Anais Brasileros de

7.-English, M. \& Atkinson, R. (1973).An improved method for the isolation of fungi in onychomycosis. Bristish Journal of Dermatology. 88: 237-241

8.- Kligma, A. (1950).Basidiomycete probably causing onychomycosis. The Journal of Investigative Dermatol.1:67-70

9.-Looder, J. (1970).General classification of yeast. In: Looder J., ed. The yeast: a taxonomic study. North-Holland Publishing Amsterdam. pp 1-33

10.- Mc Aller, R. (1981).Fungal infections of nails in Western Australia. Micopathologia. 73:115-120

11.- Negroni, P. \& Briz de Negroni, C. (1984).Agentes oportunistas de las micosis de las uñas. Revista Argentina de Micologia 7:2-4

12.-Piontelli, E .\& Toro, M.A.(1987). Comentarios biomórficos y clínicos sobre el género Fusarium. Hialohifomicosis en uñas. Boletín Micológico 3: 213-221., 1987.

13.(1988).Comentarios biomorfológicos y clínico s sobre el género Scopulariopsis.Bainier. Hialohifomicosis en uñas y piel.II. Boletin Micológico 3:259-273

14.- Prochacki, M. \& Bielunsa, S. (1967). Observations on onychomycosis. Proceedings of 2nd International Symposium on Medical Mycology. Poznan. Polonia. 81-84. Ed. W. Sowinski.

15.- Rebell,G. \& Taplin ,D.(1970).Dermatophytes-Their recognition and identification. Coral Gables, Fla. Univ. Of Miami Press.
16.- Reynolds, R. \& Braude, A. I. (1956).The filament inducing property of blood for Candidaalbicans: its nature and significance. Clinical research Proceding 4:40

17.- Restrepo, A.; Arango, M.; Velez,H.; Uribe, L. (1976).The isolation of Botryodiplodia theobromae from a nail lesion. Sabouraudia 14:1-4

18.- Reddy, B.; Singh, G.; Shorma, B. (1977).Onychomycoses and nail dystrophy. Indian Journal Dermatol. 23:1-4

19.- Rodriguez, M.; Fernández, C.; Rodríguez, R.; Martínez, G.(1993). Estudio clínico-micológico de onicomicosis en ancianos. Revista do Instituto do Medicina Tropica I do Sao Paulo 35:213-217

20.- Rosas, L. \& Zaror, L. (1987).Onicomicosis en Valdivia 1987. Seminario de Titulación. Universidad Austral de Chile.

21.- Williemsem, M. (1993).Changing patterns in superficial infections: Focus on onychomicoses. Journal of the European Academy of Dermatology and Venerology 2:6-11

22.- Zaias, N. (1972).Onychomycosis. Archives of Dermatology. 105:263-274

23.- Zaror, L. (1979). Onicomicosis en Valdivia. Revista Médica de Chile. 105:558-559

24.- Zaror, L. (1974).Dermatomicosis en el sur de Chile. Revista Médica de Chile. 102:299-300

25.- Zaror, L.; Neron, N. ; Moreno, M.; Frick, P.; Siegmund, I..; Norambuena, L. (1983).Micologia e histopatologia de la uña. Revista Argentina de Micología 6:6-13

26.-Zaror, L.; Moreno, M.; Frick, P. (1982).Micosis superficiales en Valdivia, Chile. Revista Latinoamericana de Microbiologia, 24:205-209

27.- -Zaror, L . \& Frick, P. (1980). Onicomicosis por Scopulariopsis brevicaulis. Rev. Med de Chile 108:721-722

28.--Zaror, L .; Frick, P.; Moreno, M. (1982). Micosis ungueal por Scopulariopsis brevicaulis. Revista Argentina de Micologia 5:12-15 